\title{
Exportation saisonnière d'herbicides vers les cours d'eau mesurée sur six champs agricoles sous quelques pratiques culturales du maïs (Basses-Terres du St-Laurent) Seasonal transport of herbicides to surface water as measured on six agricultural fields under different corn-cropping regimes (St-Lawrence Lowlands)
}

\author{
P. Lafrance, O. Banton et P. Gagné
}

Volume 10, numéro 4, 1997

URI : https://id.erudit.org/iderudit/705288ar

DOI : https://doi.org/10.7202/705288ar

Aller au sommaire du numéro

\section{Éditeur(s)}

Université du Québec - INRS-Eau, Terre et Environnement (INRS-ETE)

ISSN

0992-7158 (imprimé)

1718-8598 (numérique)

Découvrir la revue

Citer cet article

Lafrance, P., Banton, O. \& Gagné, P. (1997). Exportation saisonnière d'herbicides vers les cours d'eau mesurée sur six champs agricoles sous quelques pratiques culturales du maïs (Basses-Terres du St-Laurent). Revue des sciences de l'eau / Journal of Water Science, 10(4), 439-459.

https://doi.org/10.7202/705288ar
Résumé de l'article

L'identification de pratiques agricoles qui minimisent les risques de contamination des eaux de surface nécessite d'évaluer l'importance des voies de transfert des herbicides vers les cours d'eau. Le but de cette étude est d'évaluer à l'échelle du champ agricole et pour une saison de culture l'exportation effective de l'atrazine et du métolachlore par ruissellement de surface et par drainage, ceci pour des conditions pédo-climatiques et agronomiques représentatives de la culture intensive du maïs-grain dans les Basses-Terres du St-Laurent (Québec). Pour les deux premiers événements pluviaux d'importance suivant l'application des herbicides, seulement deux des six champs étudiés ont présenté un ruissellement quittant le champ : les concentrations en herbicides ont atteint $1200 \mathrm{mg} / \mathrm{L}$ et $2400 \mathrm{mg} / \mathrm{L}$. La charge exportée en herbicides semble inférieure dans le cas du non travail du sol (semis direct), comparativement au labour conventionnel. Les concentrations en herbicides dans l'eau de drainage sont inférieures à $6 \mathrm{mg} / \mathrm{L}$ (pour la majorité inférieures à $1-2 \mathrm{mg} / \mathrm{L}$ ) pour quatre champs, alors que deux champs ont présenté des concentrations atteignant 40-60 mg/L. La charge exportée par drainage apparaît être faible dans le cas de l'application d'herbicides en bandes, comparativement à l'application en surface totale. La masse en herbicides exportée par ruissellement (estimée à partir de coefficients de ruissellement probables) serait supérieure à celle par drainage. Une démarche destinée à diminuer les masses en herbicides exportées devrait ainsi viser la principale voie de cette exportation, c'est-à-dire le ruissellement de surface. 


\title{
Exportation saisonnière d'herbicides vers les cours d'eau mesurée sur six champs agricoles sous quelques pratiques culturales du maïs (Basses-Terres du St-Laurent)
}

\author{
Seasonal transport of herbicides to surface water \\ as measured on six agricultural fields under different \\ corn-cropping regimes (St-Lawrence Lowlands)
}

\section{P. LAFRANCE $^{*}, 0$. BANTON $^{\dagger}$ et P. GAGNÉ ${ }^{\dagger}$}

Reçu le 13 décembre 1996, accepté le 4 juin $1997^{* *}$.

The use of pesticides in agriculture may result in the degradation of surface water quality. Since agricultural practices affect the transport of pesticides, there is a need to identify practices which minimize the contribution of the different transport paths to the streams, i.e. runoff and drainage. The aim of this study was to evaluate at the field scale and for one growing season the transport of the herbicides atrazine and metolachlor to surface water under soil, climatic and agricultural conditions representative of those encountered for intensive corn cropping in the St-Lawrence Lowlands (Quebec).

Six agricultural fields (Fig. I) were studied in 1995. Previous agricultural practices in 1994 and soil texture are summarized in Tables 1 and 2, respectively. Conventional practices (tillage with moldboard plow and application of herbicides over the entire area of the field) and conservation practices (no-till and banded application of herbicides over the seeded row) were studied. Each field was solely and entirely drained by one subsurface drain. The commercial formulation used in 1995 contained a mass of metolachlor two times higher than that for atrazine. Herbicide concentrations in runoff and drainage waters were monitored during the two first important rainfall events that occurred after herbicide application (Table 3). Sampled runoff corresponded to the water reaching a drainage channel or a stream. Drainage water was also collected for 3.5-4.5 months following the initial application. A total of 164 water samples was obtained. After sediment removal, metolachlor, atrazine and its dealkylated metabolite deethylatrazine (DEA) were extracted using a liquid-solid extraction procedure and analyzed by gas chromatography.

Only two fields produced runoff and the concentrations of parent-compounds (Fig. 2 and 3) were high and varied during rainfall events between $60-500 \mu \mathrm{g} / \mathrm{L}$ (Field 2) or 130-2,400 $\mu \mathrm{g} / \mathrm{L}$ (Field 6). Concentrations found during the first rainfall event were higher than those encountered during the second event. The DEA/atrazine concentration ratio (DAR) was below or near 0.1, indicating runoff of recently applied atrazine (low degradation). These two fields present

1. Institut National de la Recherche Scientifique, Université du Québec, INRS-Eau, 2800 rue Einstein, C.P. 7500, Sainte-Foy, Québec, G1V 4C7, Canada.

* Auteur à qui doit être adressée la correspondance.

* * Les commentaires seront reçus jusqu'au 20 juillet 1998. 
similar soil texture, pluviometry and sampling periods after herbicide application. Based on runoff coefficients observed for other agricultural fields $(1-30 \%)$, it was estimated that the mass losses for herbicides (Table 4) would be higher under conventional tillage (Field 6) as compared to no-till (Field 2).

Significant transport of herbicides by drainage was observed during the two rainfall events. The losses of herbicides that occurred after these events and during a dry growing season (little or no drainage flow) were low. The drainage losses (concentration or masses) during the two rainfall events for Field 1 (clay) were very low. This was attributed to the low drainage capacity of the soil, to the low rainfall intensities as well as to the important delay between the initial application and the subsequent rainfalls. For silty clay loam to loam soils, the drainage flow increased in the 6-12 h period following the onset of rainfall, as did the herbicide concentrations. Metolachlor concentrations were slightly higher or close to those for atrazine: this was attributed to its possible more rapid decay and to its stronger tendency to adsorb to the soil.

During the rainfall events, four fields exhibited herbicide concentrations from drainage less than $6 \mu \mathrm{g} / \mathrm{L}$ (mostly $<1-2 \mu \mathrm{g} / \mathrm{L}$ ). Fields 2 and 6 yielded parent-compound concentrations as high as $40-60 \mu \mathrm{g} / \mathrm{L}$ (Fig. 4 and 5 ). The DAR values found for drainage water of Field $2(0.1-0.5)$ were higher than those observed from runoff, indicating signiticant dealkylation of atrazine had occurred during its transport in the unsaturated zone. Field 6 allowed the monitoring of the DAR over the growing season and an inverse relationship was found between the DAR and atrazine concentration (Fig. 6). This was attributed to the larger variation in atrazine concentration during a rainfall as compared to that of DEA. A DAR value near 1 was obtained at 1-2 months after application, indicating important degradation of atrazine.

The total mass losses of parent-compounds (two rainfall events) were evaluated (Table 5) except for Fields 2 and 4 which present frequent submerged drains. Banded herbicide application (Field 5) results in consistent lower losses of herbicide masses than those obtained for application over the entire surface (e.g. Field 5 compared to Fields 3 and 6 ). It should be noted that the higher export observed for the entire surface application may be partly attributed to a shorter delay between application and rainfalls (Fields 3 and 6 ) or to a higher rainfall intensity (Field 6).

Although runoff reaching surface waters was limited, it was estimated that the total herbicide losses (Table 4) during the two rainfall events were higher than those from drainage (Table 5). In the perspective of reducing the herbicide loads reaching streams, it appears that remedial actions should focus on this main route of transport. Thus, complementary actions such as vegetated buffer strips to intercept cropland runoff may possibly be useful to limit herbicide transfer to streams in intensive agricultural zones.

Key words : surface water, ground water: atrazine, metolachlor, transport, tillage.

L'identification de pratiques agricoles qui minimisent les risques de contamination des eaux de surface nécessite d'évaluer l'importance des voies de transfert des herbicides vers les cours d'eau. Le but de cette étude est d'évaluer à l'échelle du champ agricole et pour une saison de culture l'exportation effective de l'atrazine et du métolachlore par ruissellement de surface et par drainage, ceci pour des conditions pédo-climatiques et agronomiques représentatives de la culture intensive du naïs-grain dans les Basses-Terres du St-Laurent (Québec). Pour les deux premiers événements pluviaux d'importance suivant l'application des herbicides, seulement deux des six champs étudiés ont présenté un ruissellement quittant le champ : les concentrations en herbicides ont 
atteint $1200 \mu \mathrm{g} / \mathrm{L}$ et $2400 \mu \mathrm{g} / \mathrm{L}$. La charge exportée en herbicides semble inférieure dans le cas du non travail du sol (semis direct), comparativement au labour conventionnel. Les concentrations en herbicides dans l'eau de drainage sont inférieures à $6 \mu \mathrm{g} / \mathrm{L}$ (pour la majorité inférieures à 1-2 $\mu \mathrm{g} / \mathrm{L}$ ) pour quatre champs, alors que deux champs ont présenté des concentrations atteignant 40-60 $\mu \mathrm{g} / \mathrm{L}$. La charge exportée par drainage apparaît être faible dans le cas de l'application d'herbicides en bandes, comparativement à l'application en surface totale. La masse en herbicides exportée par ruissellement (estimée à partir de coefficients de ruissellement probables) serait supérieure à celle par drainage. Une démarche destinée à diminuer les masses en herbicides exportées devrait ainsi viser la principale voie de cette exportation, c'est-à-dire le ruissellement de surface.

Mots-clés : eaux de surface, atrazine, métolachlore, exportation, ruissellement, drainage.

\section{1 - INTRODUCTION}

L'utilisation d'herbicides dans la culture intensive du maiss est susceptible d'entraîner des impacts environnementaux préoccupants. Une étude indique que le degré de contamination, notamment par l'atrazine et le métolachlore, de cours d'eau serait proportionnel aux superficies cultivées en maïs (BERRYMAN et GIROUX, 1994). D'autres études ont montré la présence d'herbicide(s) dans des cours d'eau du Québec (FORREST et CAUX, 1990), dont certains utilisés pour la production d'eau potable (AYOTTE et LARUE, 1990). L'atrazine et le métolachlore sont parmi les herbicides les plus fréquemment détectés. Leurs concentrations peuvent dépasser le critère pour la qualité de vie aquatique, soit respectivement $2 \mu \mathrm{g} / \mathrm{L}$ et $8 \mu \mathrm{g} / \mathrm{L}$, ou la recommandation canadienne pour la somme de l'atrazine et de ses sous-produits dans l'eau potable qui est de $5 \mu \mathrm{g} / \mathrm{L}$ (BERRYMAN et GIROUX, 1994).

Le développement des pratiques culturales sur maïs devrait donc viser à concilier les objectifs de la productivité agricole avec ceux de la protection de la qualité des eaux. Parmi ces pratiques culturales, les travaux de conservation du sol (tel que le semis direct) sont reconnus pour limiter le ruissellement et l'érosion hydrique (FAWCETT et al., 1994 ; BERNARD et al., 1996) ainsi que, dans de nombreux cas, la charge en pesticides exportée vers les cours d'eau (FAWCETT et al., 1994). Cette diminution du ruissellement s'accompagne d'une infiltration d'eau plus importante dans le sol (FLURY, 1996). Par ailleurs, l'application d'herbicides en bandes sur le rang de maîs peut réduire d'au moins $50 \%$ la quantité d'herbicides utilisée, tout en permettant un désherbage approprié lorsque combinée au sarclage mécanique (EADIE et al., 1992 ; LEBLANC et al., 1995). Comparativement à une application en surface totale, une application en bandes conduit à une diminution de la concentration en atrazine dans l'eau de ruissellement ou de drainage (KAY et BAKER, 1989 ; GAYNOR et al., 1995a).

Il est reconnu que plusieurs facteurs conditionnent les quantités d'herbicide exportées par ruissellement ou par drainage, tels que : la texture et la structure du sol (FLURY, 1996), le délai entre l'application et le premier événement pluvial ainsi que l'intensité-durée de celui-ci (LEONARD, 1990 ; LAFRANCE et BANTON, 1996). Les 
pertes d'herbicides par ruissellement sont généralement inférieures à $2 \%$ de la quantité appliquée, les pertes les plus élevées étant observées lors d'un court délai entre l'application et le premier événement pluvial d'importance (CHESTERS et al., 1989 ; LAFRANCE et BANTON, 1996). Les pertes annuelles d'herbicides par drainage, qui ont fait l'objet de peu d'études, représentent généralement moins de $0,1 \%$ à $1 \%$ de la quantité appliquée (FLURY, 1996). Peu de travaux ont comparé les pertes d'herbicides dans l'eau de ruissellement et de drainage. GAYNOR et al. (1992) ont montré pour l'atrazine des pertes par ruissellement (pente $<1 \%$ ) bien supérieures aux pertes par drainage. Dans une autre étude menée sur le même sol (GAYNOR et al., 1995b), les pertes par ruissellement ont été semblables ou inférieures à celles par drainage, selon les conditions climatiques de l'année d'étude.

Au Québec, aucune étude n'a quantifié l'importance à la fois du ruissellement de surface et du drainage sur les pertes saisonnières en herbicides vers les cours d'eau. Le but de cette étude est de quantifier, pour une saison de culture, ces pertes en atrazine et en métolachlore sur six champs (Basses-Terres du St-Laurent) présentant des textures de sol et des pratiques culturales représentatives de la culture intensive de maïs-grain. Les pratiques sont : 1) labour conventionnel et pulvérisation d'herbicides en surface totale ou en bandes, ou 2) semis direct et pulvérisation en surface totale. L'étude évalue les pertes survenant lors des deux premiers (et plus importants) événements pluviaux suivant l'application. Le suivi des pertes dans l'eau de drainage a été réalisé lors des deux événements et durant la saison de croissance (transfert dans la zone non saturée du sol).

\section{2 - MATÉRIEL ET MÉTHODES}

\subsection{Pratiques agronomiques et caractéristiques des sols et des champs}

Six champs ont été sélectionnés à l'intérieur de deux régions de culture intensive du maïs-grain. Les champs sont localisés dans les municipalités de (fig. 1): Varennes (Champ 1); St-Dominique (Champ 2); St-Antoine-sur-Richelieu (Champs 3 et 4) et St-Valérien-de-Milton (Champs 5 et 6). Le tableau 1 indique les antécédents culturaux de 1994, ainsi que les pratiques culturales de la saison de suivi en 1995. L'atrazine a été utilisée avant 1994 pour tous ces champs, mais les taux d'application ne sont pas connus. En 1995, les agriculteurs ont utilisé la formulation Primextra Léger ${ }^{\mathrm{MD}}$, un mélange de métolachlore et d'atrazine en proportion $2: 1$ (330 $\mathrm{g} / \mathrm{L}$ de métolachlore, $162 \mathrm{~g} / \mathrm{L}$ d'atrazine, $8 \mathrm{~g} / \mathrm{L}$ de triazines connexes inconnues). Les caractéristiques des sols et des champs sont indiquées au tableau 2. L'analyse des sols (CPVQ, 1988), prélevés à la tarière manuelle à 0$20 \mathrm{~cm}$, a été faite en pré-semis du maïs au laboratoire du Ministère de l'Agriculture, des Pêcheries et de l'Alimentation du Québec de Rock Forest (Qué.). Chaque champ est entièrement et exclusivement drainé par un seul drain (profondeur : 1,0-1,3 $\mathrm{m}$; pente : 1-2\%). 


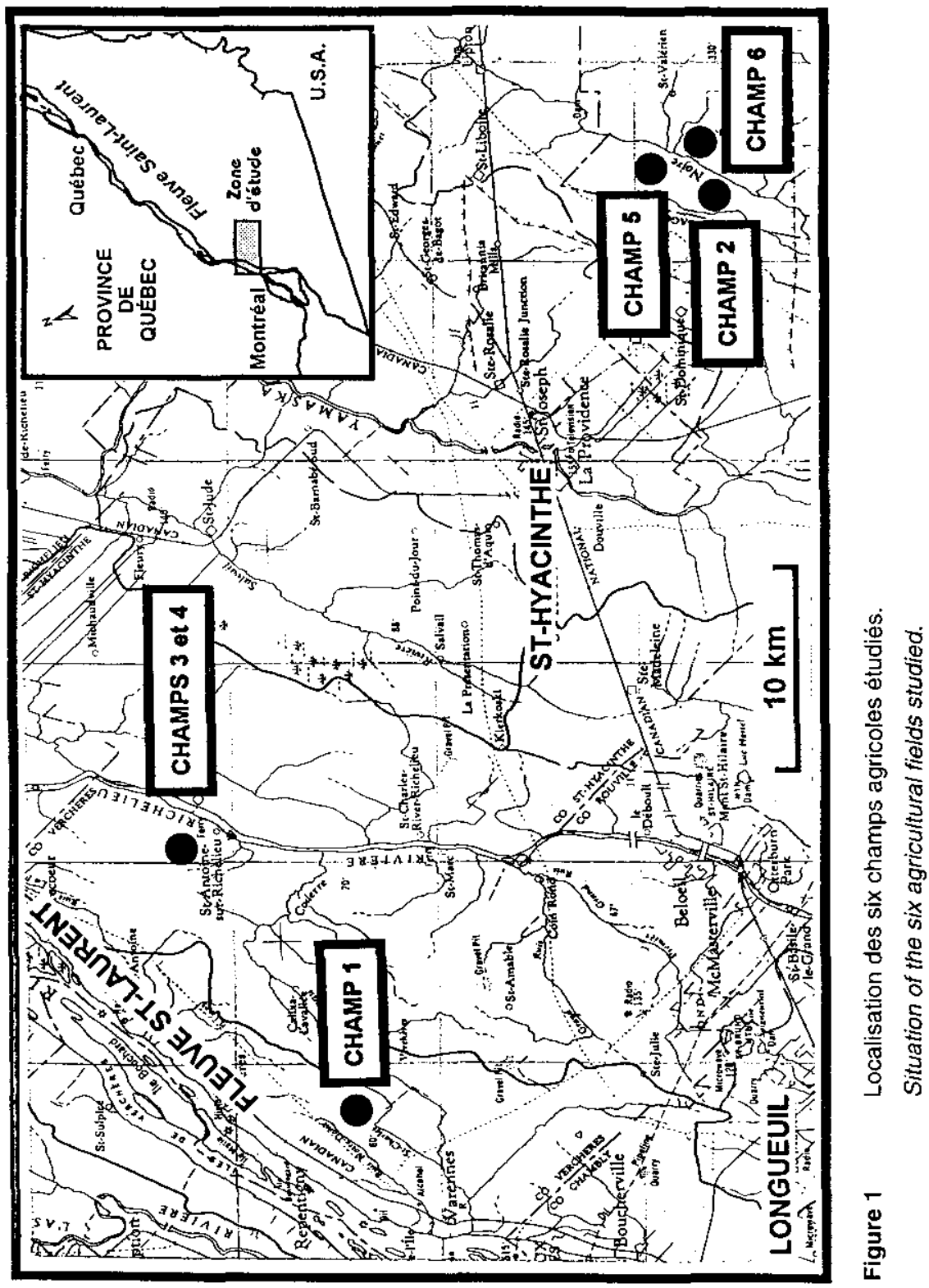


Tableau 1 Antécédents culturaux de 1994 et pratiques agronomiques en 1995.

Table1 Previous agricultural practices (1994) and practices applied in 1995.

\begin{tabular}{|c|c|c|c|c|c|c|}
\hline \multirow{2}{*}{ Activités 1994} & \multicolumn{6}{|c|}{ Champ agricole } \\
\hline & 1 & 2 & 3 & 4 & 5 & 6 \\
\hline Culture & Orge & Soya & Haricots secs & Haricots secs & Mails-grain & Maïs-grain \\
\hline Travail du sol & Semis direct & Semis direct & Laboura $^{\mathrm{a}}$ & Labour & Labour & Labour \\
\hline Pesticides & $\begin{array}{l}\text { Bromoxynil et } \\
\text { MCPA }\left(T^{\mathrm{b}}\right)\end{array}$ & $\begin{array}{l}\text { Imazétaphir et } \\
\text { Métribuzine (T) }\end{array}$ & Bentazone $(T)$ & Bentazone $(T)$ & $\begin{array}{l}\text { Diméthénamide } \\
\left(\mathrm{B}^{\mathrm{b}} \text { de } 40 \mathrm{~cm}\right)\end{array}$ & $\begin{array}{l}\text { Diméthénamide } \\
\text { (B de } 40 \mathrm{~cm} \text { ) }\end{array}$ \\
\hline \multicolumn{7}{|l|}{ Activités 1995} \\
\hline Travail du sol & Semis direct & Semis direct & Hersage $^{\mathrm{a}}$ & Hersage & Hersage & Hersage \\
\hline Semis du mais & 10 mai & $7 \mathrm{mai}$ & $9 \mathrm{mai}$ & $9 \mathrm{mai}$ & $8 \mathrm{mai}$ & $7 \mathrm{mai}$ \\
\hline Herbicides & 7 juin $\left(\right.$ Post $\left.^{c}\right)$ & 23 mai (Préc) & 13 mai (Pré) & 9 mai (Pré) & 8 mai (Pré) & 26 mai (Post) \\
\hline Application & Surface totate & Surface totale & Surface totale & Bandes $38 \mathrm{~cm}$ & Bandes $38 \mathrm{~cm}$ & Surface totale \\
\hline Formulation & $7,5 \mathrm{~L} / \mathrm{ha}$ & 7,0 L/ha & $7,5 \mathrm{~L} / \mathrm{ha}$ & $6,8 \mathrm{~L} / \mathrm{ha}$ & $7,5 \mathrm{~L} / \mathrm{ha}$ & $7,0 \mathrm{~L} / \mathrm{ha}$ \\
\hline Atrazine $^{d}$ & $1,21 \mathrm{~kg} / \mathrm{ha}$ & $1,13 \mathrm{~kg} / \mathrm{ha}$ & $1,21 \mathrm{~kg} / \mathrm{ha}$ & $1,10 \mathrm{~kg} / \mathrm{ha}$ & $1,21 \mathrm{~kg} / \mathrm{ha}$ & $1,19 \mathrm{~kg} / \mathrm{ha}$ \\
\hline Métolachlored & $2,47 \mathrm{~kg} / \mathrm{ha}$ & $2,31 \mathrm{~kg} / \mathrm{ha}$ & $2,47 \mathrm{~kg} / \mathrm{ha}$ & $2,24 \mathrm{~kg} / \mathrm{ha}$ & $2,47 \mathrm{~kg} / \mathrm{ha}$ & $2,31 \mathrm{~kg} / \mathrm{ha}$ \\
\hline Sarclage & - & - & - & 9 juin & 6 juin & - \\
\hline
\end{tabular}

a Le labour a été réalisé à l'automne 1994. Le hersage en 1995 est fait pour la préparation du lit de semence.

† $T$ : application en surface totale; $B$ : application en bandes.

c Post : application en postievée du maïs-grain ; Pré : application en prélevée du maïs-grain.

- Taux d'application de matières actives. 
Tableau 2 Caractéristiques des sols et des champs étudiés.

Table 2 Characteristics of the studied soils and fields.

\begin{tabular}{|c|c|c|c|c|c|c|}
\hline \multirow{2}{*}{ Sol } & \multicolumn{6}{|c|}{ Champ agricole } \\
\hline & 1 & 2 & 3 & 4 & 5 & 6 \\
\hline pHàl'eau & 6,9 & 6,3 & 6,1 & 6,3 & 6,0 & 5,4 \\
\hline Matières org. $(\%)$ & 2,8 & 2,4 & 3,7 & 4,5 & 4,2 & 7,3 \\
\hline Sable $(\%)$ & 24 & 12 & 38 & 32 & 36 & 16 \\
\hline $\operatorname{Limon}(\%)$ & 24 & 54 & 36 & 40 & 46 & 56 \\
\hline Argile $(\%)$ & 52 & 34 & 26 & 28 & 18 & 28 \\
\hline Texture & A & LLIA & $L$ & LA & L & LLIA \\
\hline \multicolumn{7}{|l|}{ Champ } \\
\hline Superficie (ha) & 1,2 & 6,0 & 4,0 & 4,0 & 9,0 & 8,0 \\
\hline Pente $(\%)$ & $<1$ & $<1$ & $<1$ & $<1$ & $1-3$ & $1-3$ \\
\hline Drainage naturel & Mauvais & Imparfait & Mauvais & Mauvais & Imparfait & Imparfait \\
\hline $\begin{array}{l}\text { Cours d'eau } \\
\text { récepteur }\end{array}$ & Tributaire du St-Lâurent & $\begin{array}{c}\text { Fossé - ruisseau } \\
\text { des Auinages }\end{array}$ & Tributaire du Richelieu & Tributaire du Richelieu & Ruisseau des Aulnages & Fossé - rivière Noire \\
\hline
\end{tabular}

a A : argile, série St-Urbain ; LA : loam argileux, série Bouchenille ; LLiA : loam limono-argileux, série Yamaska ; L : loam. Textures selon la ciassification du CEPPAC (1987). Séries de sol selon CANN et al. (1947) ainsi que LAMONTAGNE et NOLIN (1990). 


\subsection{Pluviométrie et échantillonnage d'eau de ruissellement et de drainage}

Toutes les précipitations supérieures à 2-3 mm après l'application ont été suivies sur le terrain. L'échantillonnage de l'eau de ruissellement quittant le champ a été effectué durant les deux premiers événements pluviaux d'importance. La hauteur d'eau a été mesurée à l'aide d'un pluviomètre lors de chaque échantillonnage (tabl. 3). Pour la majorité des champs, l'échantillonnage a eu lieu dans les deux premières semaines suivant l'application des herbicides, sauf pour le Champ 1 (période de plus d'un mois).

Tableau 3 Hauteurs d'eau précipitée (pour les deux premiers événements pluviaux) et périodes d'échantillonnage.

Table 3 Precipitations (for the two first important rainfall events that followed herbicide application) and sampling periods.

\begin{tabular}{|c|c|c|c|c|c|c|}
\hline \multirow{2}{*}{$\begin{array}{l}\text { Hauteur d'eau } \\
\text { précipitée (mm) }\end{array}$} & \multicolumn{6}{|c|}{ Champ agricole } \\
\hline & 1 & 2 & 3 & 4 & 5 & 6 \\
\hline Événement $1 / 2$ & $8 / 9$ & $20 / 33$ & $21 / 16$ & $21 / 16$ & $21 / 13$ & $16 / 30$ \\
\hline $\begin{array}{l}\text { Total } \\
\text { Échantillonnage }\end{array}$ & 17 & 53 & 37 & 37 & 34 & 46 \\
\hline Événement 1 & $30-31$ & $6-7$ & $1-2$ & $6-7$ & $7-8$ & $3-4$ \\
\hline Événement 2 & $37-38$ & $10-12$ & $3-4$ & $8-9$ & $9-10$ & $8-9$ \\
\hline Saison (drainage) & $39-101$ & $13 \cdot 116$ & $5 \cdot 125$ & $10-130$ & $11-131$ & $10-113$ \\
\hline
\end{tabular}

${ }^{3}$ Période de prélèvement (jours après l'application des herbicides). Le drainage a été suivi durant la saison après les deux èvénements.

L'eau de ruissellement quittant le champ a été recueillie (intervalles d'environ 1,0-1,5 h) en bordure du champ dans un récipient métallique, puis transvasée dans une flacon de $1 \mathrm{~L}$ en polyéthylène haute densité (PEHD), matériel très peu adsorbant pour les herbicides étudiés (TOPP et SMITH, 1992). Pour l'eau de drainage, un suivi de 3,5 à 4,5 mois (selon les dates d'application des herbicides) a. été fait. L'eau a d'abord été échantillonnée durant les deux premiers événements suivis (intervalles d'environ 5-6 h), puis un suivira été fait tous les dix jours jusqu'à la mi-juillet, et finalement tous les quinze jours jusqu'à la mi-septembre. Après la mi-juin, les drains suivis n'ont pas généré d'eau de drainage dans $55 \%$ des cas (période de faible pluviométrie). Pour les drains non submergés, le débit a été estimé lors des prélèvements (flacon de $1 \mathrm{~L}$ en PEHD). Pour les drains partiellement submergés, une méthode par succion a permis de prélever l'eau à l'intérieur du drain. Une extrémité d'un tuyau en cuivre était placée de $30 \mathrm{~cm}$ à $60 \mathrm{~cm}$ à Iintérieur du drain tandis que l'autre extrémité était reliée à un flacon de $1 \mathrm{~L}$ (PEHD) : une pompe reliée au flacon a permis d'aspirer l'eau du drain dans celuici. Ce prélèvement a été réalisé en un point intérieur du drain plus élevé que le plan d'eau submergeant l'extrémité du drain : ainsi, seule l'eau de drainage a été prélevée (pas de mélange). Un total de 164 échantillons a été obtenu. Les sédiments ont été éliminés par filtration sur microfibres de verre $(1,5 \mu \mathrm{m})$ puis sur nylon-66 $(0,45 \mu \mathrm{m})$. Les filtrats ont été entreposés à $-20^{\circ} \mathrm{C}$ (flacons en PEHD). 


\subsection{Extraction et dosage des composés herbicides}

Les composés dosés étaient le métolachlore, l'atrazine et le dééthyl-atrazine (DEA), l'un des sous-produits majeurs de déalkylation de l'atrazine (ADAMS et THURMAN, 1991). Notons que le rapport des concentrations DEA/atrazine (RDA) constitue un indicateur du temps de séjour du composé-parent dans le sol, et donc de la voie d'exportation vers les eaux de surface, i.e du ruissellement de surface versus le transfert en zone non saturée et l'exportation par te drainage (ADAMS et THURMAN, 1991 ; THURMAN et al., 1992).

Les composés herbicides ont été adsorbés sur une cartouche octadécyle (Seppak Plus C-18, Environmental Cartridge, $840 \mathrm{mg}$, Waters) et ont été élués avec de l'acétate d'éthyle saturée en eau. L'extrait a été évaporé sous jet d'azote (facteur de concentration de 250 ). Le dosage a été réalisé à l'aide d'un chromatographe en phase gazeuse (Varian, modèle 3400) avec deux détecteurs thermo-ioniques (NPD). La séparation a été réalisée à l'aide d'une colonne d'analyse DB5 et d'une colonne de confirmation DB1701 (J \& W Sci., $30 \mathrm{~m} \times 0,25 \mathrm{~mm}$ d.i. avec un film de $0,25 \mu \mathrm{m}$ ). Le gaz vecteur a été l'hélium. Un contrôle de qualité (blancs de méthode, échantillons de contrôle, fortifiés et analysés en double) a permis de s'assurer de la bonne performance de la méthode. Tous les échantillons ont reçu un étalon d'extraction (propoxure ou terbutryne) et un étalon d'injection (amétryne). La limite de quantification de la méthode (plus faible concentration dans l'eau qui est toujours détectée et correctement quantifiée) a été de $0,4 \mu \mathrm{g} / \mathrm{L}$ pour le métolachlore, de $0,1 \mu \mathrm{g} / \mathrm{L}$ pour l'atrazine et de $0,5 \mu \mathrm{g} / \mathrm{L}$ pour le DEA. Les rendements d'extraction ont été supérieurs à $90 \%$ pour l'atrazine et le métolachlore et à $60 \%$ pour le DEA. Les résultats ont été corrigés individuellement pour les rendements obtenus.

\section{3 - RÉSULTATS ET DISCUSSION}

\subsection{Eau de ruissellement}

Un nombre limité d'échantillons d'eau de ruissellement a été obtenu suite aux deux événements pluviaux suivis. Ceci a été attribué à la faible pente des champs, à une faible pluviométrie à partir de la mi-juin et à une capacité de rétention élevée en période de déficit en eau (après la mi-jüin). Seuls des événements de plus de $15 \mathrm{~mm}$ ont conduit à un ruissellement (total de 34 échantillons), et ceci seulement pour le Champ 2 (deux points de prélèvement nommés $P 1$ et $P 2$ ) et le Champ 6 (cinq points nommés $P 1$ à $P 5$ ).

\subsubsection{Concentrations en composés herbicides}

Les figures 2 (Champ 2) et 3 (Champ 6) présentent les précipitations cumulées et les concentrations en composés herbicides retrouvées durant les deux événements pluviaux. Pour le Champ 2, les concentrations en composés-parents ont été plus élevées lors du premier événement pluvial (300-500 $\mu \mathrm{g} / \mathrm{L}$ ) que lors du second événement $(60-400 \mu \mathrm{g} / \mathrm{L})$. Le métolachlore se retrouve en concentrations soit supérieures, soit voisines de celles en atrazine : ceci est attribuable à la plus grande quantité appliquée comparativement à celle d'atrazine. Le rapport moyen des concentrations DEA/atrazine (RDA) est d'environ $0,1 \quad(0,03$ à 0,13 ) 

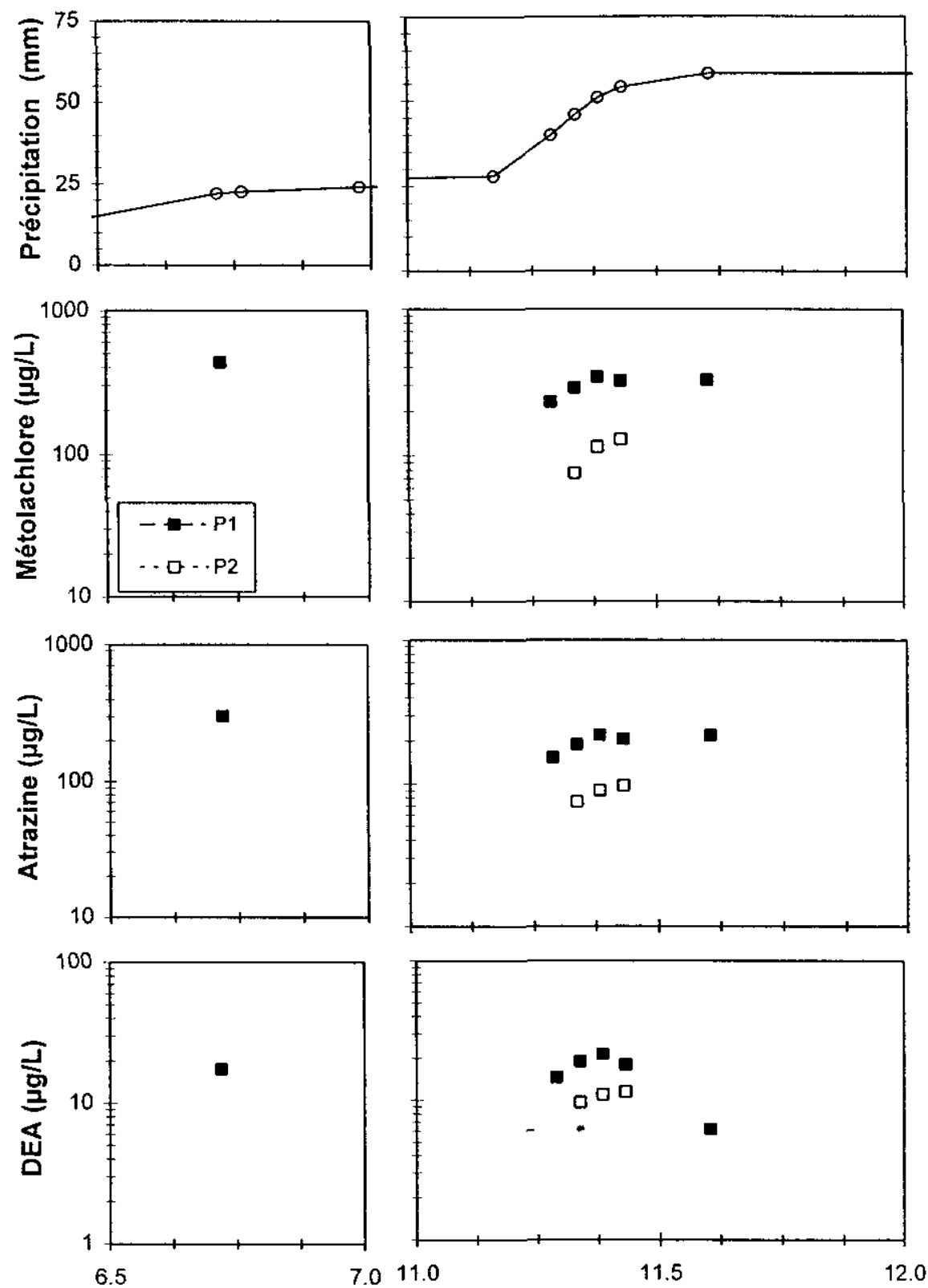

Jour après l'application des herbicides (Jour $0=23$ mai)

Figure 2 Précipitation cumulée et concentrations en composés herbicides dans l'eau de ruissellement du Champ 2. Les deux points de prélèvement sont notés en $\mathrm{P} 1$ et $\mathrm{P} 2$.

Cumulative precipitation and concentrations of herbicides in runoff water at Field 2. The two sampling locations are noted $P 1$ and $P 2$. 

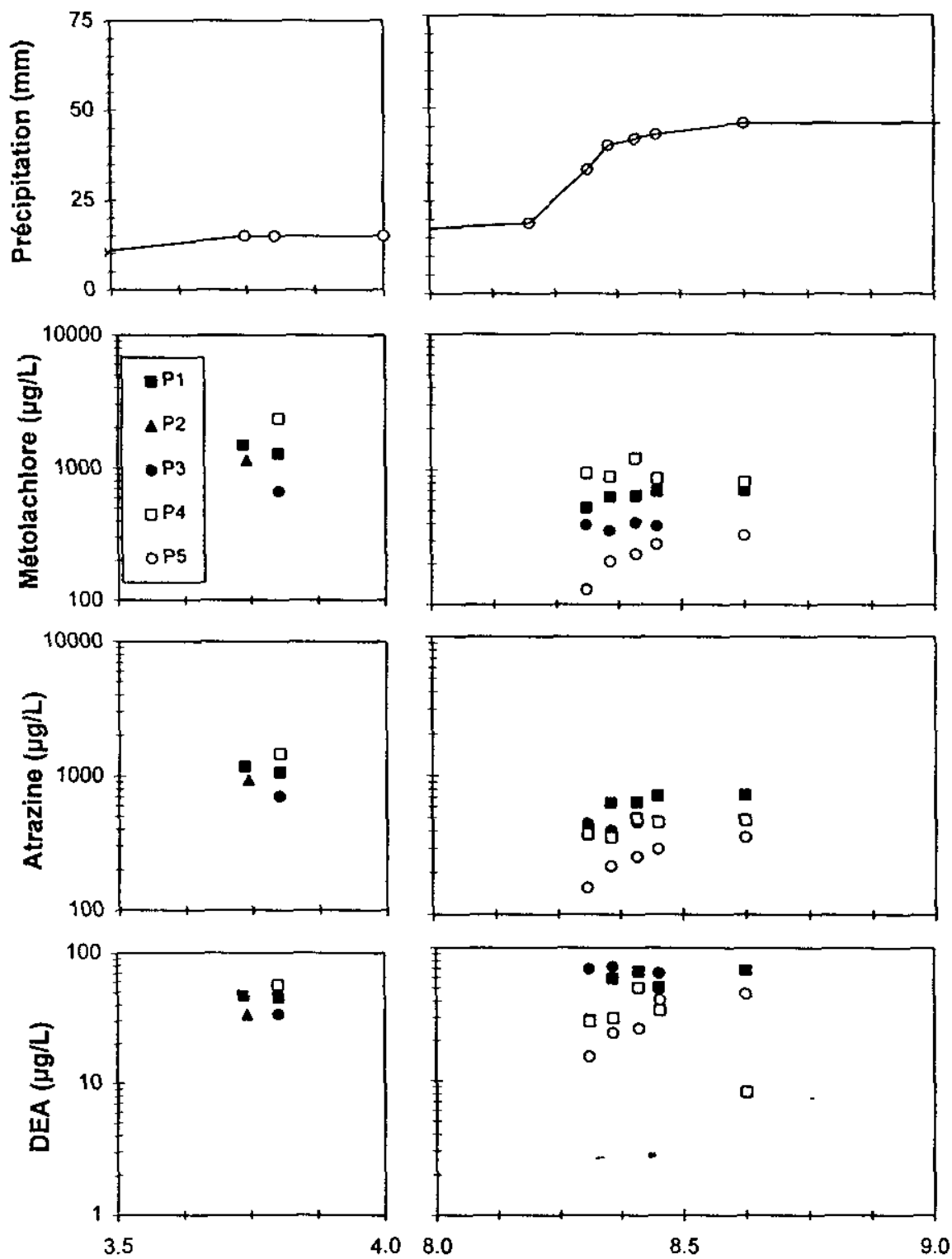

Jour après l'application des herbicides (Jour $0=26$ mai)

Figure 3 Précipitation cumulée et concentrations en composés herbicides dans reau de ruissellement du Champ 6 . Les cinq points de prélèvement sont notés P1 à P5.

Cumulative precipitation and concentrations of herbicides in runoff water at Field 6. The five sampling locations are noted P1 to PS. 
entre une et deux semaines suivant l'application. Pour le Champ 6, les concentrations obtenues en composés-parents lors du premier événement $(670-2400 \mu \mathrm{g} / \mathrm{L})$ ont été bien supérieures à celles du second événement (130-1 $200 \mu \mathrm{g} / \mathrm{L})$. Les concentrations en métolachlore sont plus élevées que celles en atrazine. L'augmentation du RDA entre le premier événement $(0,04-0,07)$ et le second $(0,02-$ 0,18 ) indique qu'une fraction significative de DEA est apparue en cinq jours. Les concentrations retrouvées dans le cours d'eau récepteur et à proximité du déversement de l'eau de ruissellement (premier événement), sont de $22 \mu \mathrm{g} / \mathrm{L}$ pour le métolachlore et $7 \mu \mathrm{g} / \mathrm{L}$ pour l'atrazine (non présentées à la fig. 3). Ces concentrations en composés-parents dépassent les critères canadiens pour la qualité de la vie aquatique. Le RDA observé dans le ruisseau est faible $(0,07)$ et est attribué à une déalkylation limitée de l'atrazine trois jours après l'application.

\subsubsection{Masse potentielle exportée en composés herbicides}

If n'a pas été techniquement possible de mesurer le volume d'eau ruisselée quittant le champ. Toutefois, et à titre d'approximation, on peut considérer que le coefficient de ruissellement serait compris dans une gamme de valeurs mesurées pour d'autres champs cultivés du Québec, soit de $1 \%$ à $30 \%$ (PESANT et al., 1987 ; LAFRANCE et al., 1996). En utilisant ces coefficients, les hauteurs d'eau précipitée ainsi que les bornes inférieures et supérieures des domaines de concentrations mesurées, il est possible d'évaluer la masse d'herbicides exportée (en g/ha) et la fraction exportée de la quantité d'herbicide appliquée, ceci pour chacun des événements pluviaux (tabl. 4). Pour les deux champs, la masse de

Tableau 4 Estimationa des masses de composés herbicides exportées par l'eau de ruissellement lors des deux événements pluviaux aux Champs 2 et 6.

Table 4 Evaluation of the masses of herbicides losses from runoff for each one of the two rainfall events at Fields 2 and 6.

\begin{tabular}{|cccccc|}
\hline \multirow{2}{*}{$\begin{array}{c}\text { Exportation des } \\
\text { composés herbicides }\end{array}$} & \multicolumn{2}{c}{ Champ 2 } & \multicolumn{2}{c|}{ Champ 6 } \\
\cline { 3 - 6 } & Pluie 1 & Pluie 2 & Pluie 1 & Pluie 2 \\
\hline $\begin{array}{c}\text { Concentration } \\
\text { dans l'eau } \\
\text { de ruissellement }(\mu \mathrm{g} / \mathrm{L})\end{array}$ & Métolachlore & $400-500^{\mathrm{b}}$ & $70-400$ & $600-1200^{\mathrm{C}}$ & $100-1100$ \\
& Atrazine & $200-400$ & $60-300$ & $600-1100$ & $100-900$ \\
$\begin{array}{c}\text { Masse exportée } \\
(\mathrm{g} / \mathrm{ha})^{\mathrm{d}}\end{array}$ & DEA & $10-20$ & $5-30$ & $20-70$ & $6-80$ \\
& Métolachlore & $0,8-29$ & $0,2-40$ & $1,0-58$ & $0,3-99$ \\
& Atrazine & $0,4-23$ & $0,2-30$ & $1,0-53$ & $0,3-81$ \\
Fraction exportée & DEA & $0,02-1,0$ & $0,02-3,0$ & $0,03-3,0$ & $0,02-7,0$ \\
$(\%)^{e}$ & Métolachiore & $0,03-1,27$ & $0,01-1,71$ & $0,05-2,91$ & $0,02-5,0$ \\
& Atrazine & $0,03-2,07$ & $0,02-2,63$ & $0,10-5,45$ & $0,03-8,36$ \\
\hline
\end{tabular}

a Basée sur des coefficients de ruissellement de $1 \%-30 \%$ mesurés ailleurs au Québec.

b Domaine de variation des concentrations durant l'événement pluviał.

c Excluant une valeur extrême de $2400 \mu \mathrm{g} / \mathrm{L}$ à un point d'échantillonnage (P4).

d Précipitation $x$ coefficient de ruissellement $x$ concentration.

e Masse exportée/masse appliquée (en \%).

métolachlore exportée est voisine ou légèrement supérieure à celle de l'atrazine, alors que la fraction exportée pour le métolachlore est la plus faible. L'exportation des herbicides peut être comparée entre les deux champs (application en surface 
totale) qui présentent des conditions pédo-climatiques peu différentes (tabl. 3). Bien que les charges réelles exportées demeurent conditionnelles aux coefficients de ruissellement réels des champs, les résultats suggèrent que la charge potentielle serait nettement supérieure pour le Champ 6 (labour) comparativement au Champ 2 (semis direct). Pour les fractions exportées, les pourcentages seraient de 1,5 à 3 fois supérieurs (selon l'herbicide et l'événement considéré) pour le labour comparativement au semis direct. Ce résultat avait rarement ou jamais été observé à l'échelle de champs agricoles.

\subsection{Eau de drainage}

\subsubsection{Concentrations en composés herbicides}

Le suivi a permis de prélever 130 échantillons d'eau de drainage. Un prélèvement par champ a été effectué (4 mai 1995) avant l'application des herbicides. Les concentrations obtenues ont été pour la majorité inférieures à la limite de quantification de la méthode. Le suivi de l'eau de drainage a été effectué durant les deux événements pluviaux et durant la saison de croissance. Après les deux événements (voir tabl. 3 pour les périodes suivies), les Champs 1 à 4 ont montré des concentrations inférieures à $0,7 \mu \mathrm{g} / \mathrm{L}$. Le Champ 5 a présenté des concentrations inférieures à $7 \mu \mathrm{g} / \mathrm{L}$ (jour 24) et à $1 \mu \mathrm{g} / \mathrm{L}$ (autres jours). Pour le Champ 6, les concentrations ont été inférieures à $10 \mu \mathrm{g} / \mathrm{L}$ (jour 13) et à $3 \mu \mathrm{g} / \mathrm{L}$ (autres jours). Les concentrations observées après les deux premiers événements pluviaux sont faibles. Considérant de plus le très faible débit de drainage observé durant cette période, les charges exportées par drainage sont considérées comme minimes.

Durant les deux premiers événements pluviaux, les concentrations mesurées pour le Champ 1 sont quasi nulles $(<0,6 \mu \mathrm{g} / \mathrm{L})$. Ceci peut être attribué à trois causes: 1) les hauteurs d'eau précipitée sont faibles ; 2) les événements sont survenus plus d'un mois après l'application, et: 3 ) le drainage a été limité en période de faible pluviométrie, malgré la présence de carbonates dans cette argile d'origine marine qui favorise une bonne conductivité hydraulique. Pour le Champ 2 (fig. 4), on observe une remontée des concentrations en composésparents jusqu'à 10-40 $\mu \mathrm{g} / \mathrm{L}$. Ceci serait dû à : 1) un délai relativement court entre l'application et les événements ; 2) une importante hauteur d'eau totale précipitée, et : 3) un drainage relativement efficace pour ce loam limono-argileux sous semis direct (possible infiltration préférentielle). Le RDA qui est faible $(0,12-0,21)$ lors de l'apparition du pic de concentration en atrazine durant un événement, augmente $(0,25-0,45)$ à la fin des événements en présence de faibles concentrations en atrazine. Dans le cas des Champs 3 et 4 qui sont adjacents, les deux échantillonnages ont eu lieu très peu de temps après l'application et les hauteurs d'eau précipitée sont identiques. La quasi-totalité des concentrations ont été inférieures à 1-2 $\mu \mathrm{g} / \mathrm{L}$ pour ces deux champs. Les concentrations maximales en composés-parents sont très légèrement plus élevées pour le Champ 3 (4-6 $\mu \mathrm{g} / \mathrm{L}$ ), sous pulvérisation en surface totale, comparativement au Champ $4(2-4 \mu \mathrm{g} / \mathrm{L})$, sous pulvérisation en bandes.

Dans le cas des Champs 5 et $\mathbf{6}$, les échantillonnages ont eu lieu peu de temps après l'application. Pour le Champ 5, les deux événements conduisent à des concentrations inférieures à $4 \mu \mathrm{g} / \mathrm{L}$. Le RDA (jour 10) a été de 0,27 et 0,33. Pour le Champ 6 (fig. 5), on note pour les deux événements une remontée des concentrations en composés-parents jusqu'à 40-54 $\mu \mathrm{g} / \mathrm{L}$. Pour ces deux champs qui sont soumis au labour, les concentrations les plus élevées sont obtenues pour 

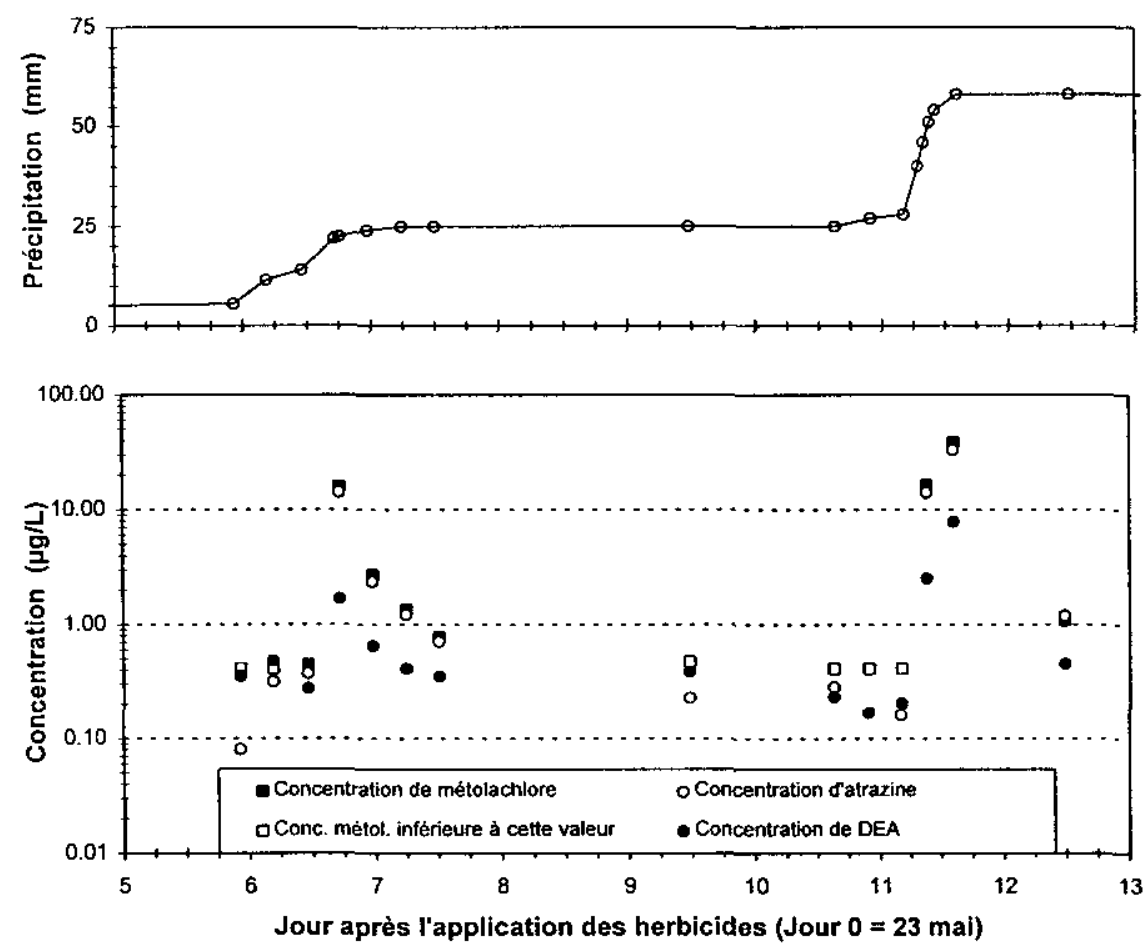

Figure 4 Précipitation cumulée et concentrations en composés herbicides dans l'eau de drainage du Champ 2.

Cumulative precipitation and concentrations of herbicides in drainage water at Field 2.

le Champ 6, sous pulvérisation en surface totale, comparativement au Champ 5 , sous pulvérisation en bandes. Cette importante différence pourrait être attribuée à la différence entre les quantités d'herbicides appliquées pour ces champs. De plus, la pluviométrie plus importante au Champ 6 a pu favoriser l'infiltration des composés-parents. Par ailleurs, le sol du Champ 6 présente un contenu élevé en matières organiques qui implique une adsorption accrue des herbicides et qui pourrait être un signe de mauvais drainage. Malgré cela, le Champ 6 a généralement présenté des concentrations supérieures à celles du Champ 2 (fig. 4 et 5) dont le sol, plus pauvre en matières organiques, retient moins les herbicides. Ceci montrerait l'importance du délai entre l'application et les événements qui est notablement plus court pour le Champ 6.

La totalité de la période suivie pour le Champ 6 permet de mettre nettement en évidence la relation inverse entre le RDA et la concentration en atrazine (fig. 6). Ceci avait déjà été observé pour le Champ 2. Pour le Champ 6, l'augmentation de la concentration en atrazine durant chacun des événements pluviaux a été de 88 et de 35 fois, alors que l'augmentation de la concentration en DEA n'a été respectivement que de 6 et de 16 fois. L'augmentation plus importante de la concentration en atrazine conduit donc à une faible valeur du RDA. 

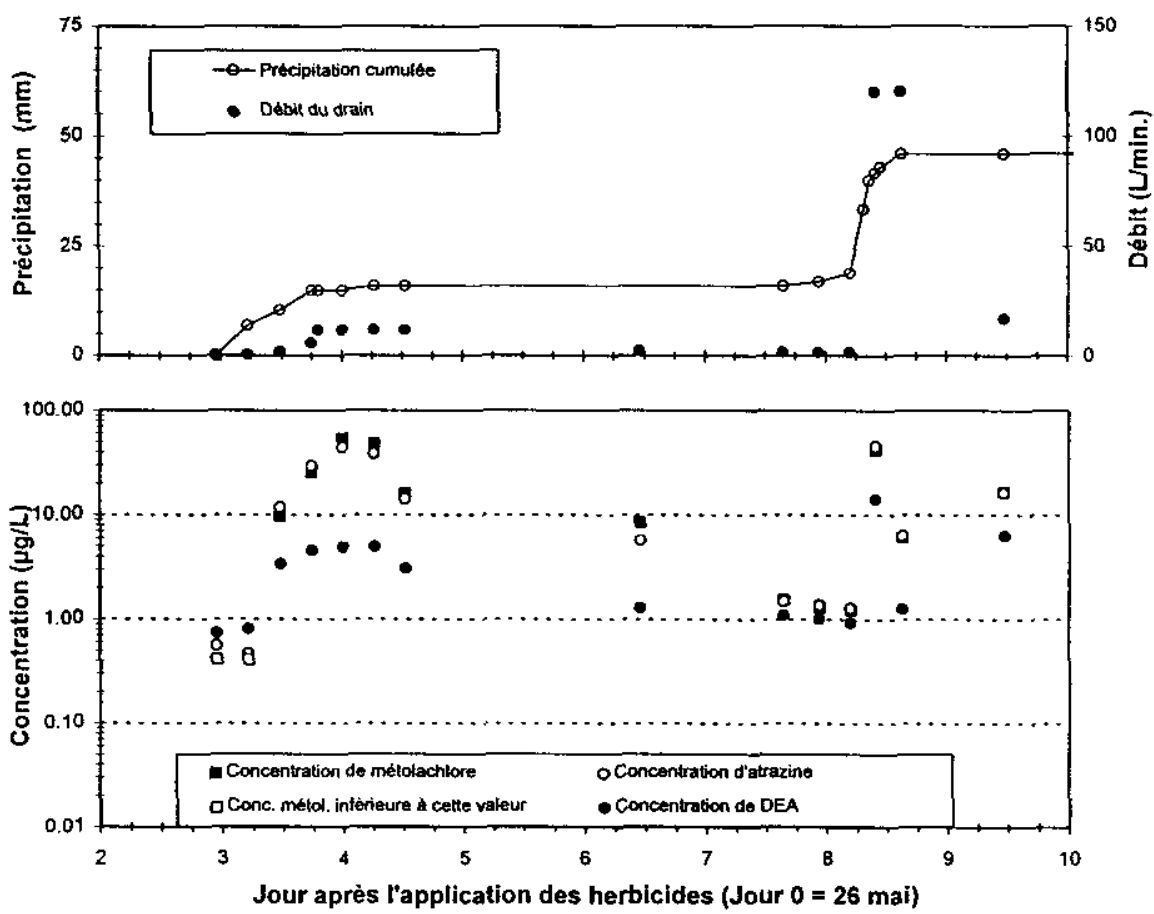

Figure 5 Précipitation cumulée, débit du drain et concentrations en composés herbicides dans l'eau de drainage du Champ 6.

Cumulative precipitation, drainage flow and concentrations of herbicides in drainage water at Field 6.

Inversement, les valeurs maximales du RDA $(0,70-0,73)$ sont obtenues entre les deux événements, en présence de faibles concentrations en atrazine qui sont alors voisines de celles en DEA. Le RDA a été environ trois fois plus élevé pour le second événement : ceci serait dû à la déalkylation de l'atrazine dans l'intervalle de 4,4 jours entre les pics des deux événements. Après ces événements, le RDA a augmenté de 0,40 à 0,94 en 16 jours puis est demeuré stable $(0,85)$ durant 42 jours. Cette valeur de RDA traduit l'importante déḡradătion de l'atrazine lors de son lent transfert en zone non saturée.

\subsubsection{Masses exportées pour chacun des événements pluviaux}

Dans le cas des Champs 2 et $\mathbf{4}$, la sortie du drain émergeait fréquemment dans l'eau du cours d'eau (tuyau submergé) : le débit ainsi que les masses d'herbicides n'ont donc pu être estimés. Pour les autres champs, les masses exportées (en $\mathrm{mg} / \mathrm{ha}$ ) ont été évaluées à partir du débit et de la concentration en composés herbicides. Pour le Champ 1, les masses exportées en composés-parents sont faibles $(<0,5 \mathrm{mg} / \mathrm{ha})$. Ceci est notamment attribué à la faible quantité d'eau drainée $(<0,2 \mathrm{~mm})$ et au long délai entre l'application et les événements. En effet, la masse de DEA exportée lors des événements (entre 0,3 et $0,5 \mathrm{mg} / \mathrm{ha}$ ) apparaît être supérieure à celle de l'atrazine. Pour le Champ 3, les masses exportées lors 


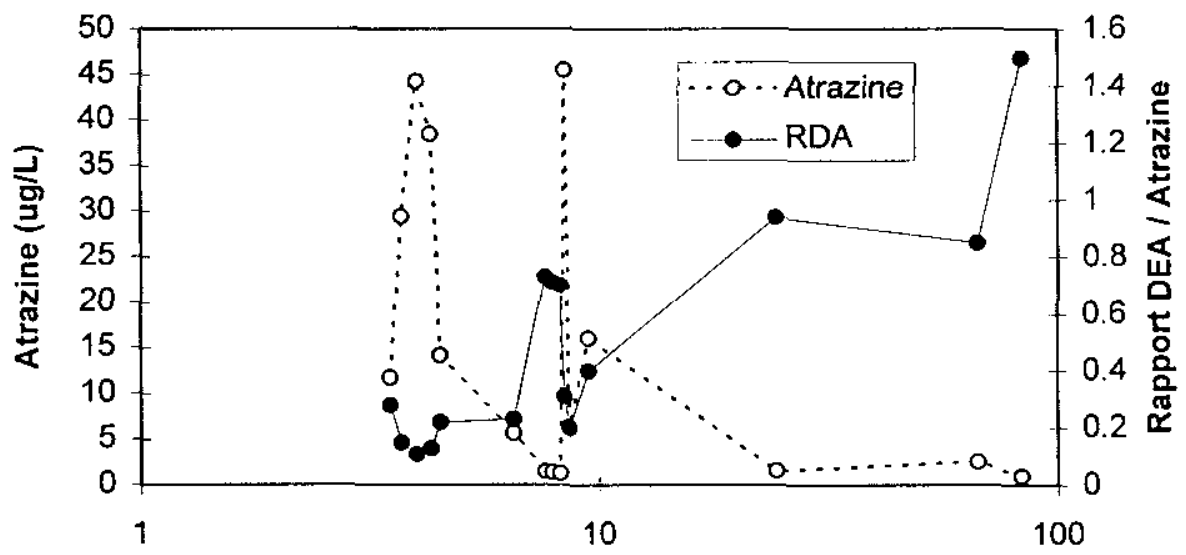

Jour suivant l'application des herbicides

Figure 6 Évolution temporelle des concentrations en atrazine et du rapport des concentrations DEA atrazine (RDA) dans leau de drainage du Champ 6. L'échelle des jours (abscisse) est logarithmique.

Temporal variation of atrazine concentration and DEA/atrazine ratio (DAR) in drainage water at Field 6 . The scale for days (abscissa) is logarithmic.

de chacun des deux événements sont respectivement de $2-14 \mathrm{mg} / \mathrm{ha}$ puis de 30 $160 \mathrm{mg} / \mathrm{ha}$ pour les composés-parents, et de $1-7 \mathrm{mg} / \mathrm{ha}$ puis de $2-13 \mathrm{mg} / \mathrm{ha}$ pour le DEA. Le second événement a généré une hauteur d'eau drainée $(3,0-10 \mathrm{~mm})$ bien supérieure après le premier qui a augmenté le contenu en eau du sol et permis l'infiltration préalable d'herbicides. Ceci expliquerait les masses plus importantes exportées durant ce deuxième événement.

Pour le Champ 5, le premier événement a généré une faible hauteur d'eau drainée $(0,5-1,3 \mathrm{~mm})$; les masses exportées sont inférieures à $3 \mathrm{mg} / \mathrm{ha}$ pour les composés-parents et à $5 \mathrm{mg} /$ ha pour le DEA. Le deuxième événement a conduit à une hauteur d'eau drainée plus importante $(1,3-3,7 \mathrm{~mm})$ : les masses exportées en composés-parents sont alors de 10-60 mg/ha et celles en DEA de 5-24 mg/ha. Pour le Champ 6, le premier événement a montré une faible hauteur d'eau drainée $(<0,5 \mathrm{~mm})$ : les masses exportées ont été de $60-140 \mathrm{mg} / \mathrm{ha}$ pour les composésparents et de 9-21 mg/ha pour le DEA. Le second événement montre une hauteur d'eau drainée plus importante $(1,4-3,7 \mathrm{~mm})$ : les masses exportées atteignent alors $180-680 \mathrm{mg} / \mathrm{ha}$ pour les composés-parents et $50-200 \mathrm{mg} /$ ha pour le DEA. Pour les Champs 5 et 6 , la masse de métolachlore est voisine de celle d'atrazine. La hauteur d'eau drainée apparaît jouer un rôle important sur l'exportation.

\subsubsection{Masses totales exportées pour les deux événements pluviaux}

La hauteur totale d'eau drainée, la masse totale d'herbicides (en $\mathrm{mg} / \mathrm{ha}$ ) ainsi que la fraction d'herbicide exportée par les deux événements pluviaux sont indiquées au tableau 5. Les intervalles de valeurs représentent les imprécisions liées aux mesures du débit et de la concentration. Déterminées à partir des résultats du contrôle de qualité (duplicata d'analyse), les erreurs sur les concentrations sont de $10 \%, 15 \%$ et $20 \%$, respectivement pour le métolachlore, l'atrazine et le 
DEA. L'erreur sur la mesure du volume d'eau drainée affecte les valeurs de la hauteur d'eau drainée (volume/surface du champ), celles du débit et donc celles de la masse exportée. Selon la technique de mesure utilisée (temps requis pour obtenir $1 \mathrm{~L}$ ), cette erreur a été estimée à $10 \%$ et jusqu'à $50 \%$ pour des débits respectivement inférieurs et supérieurs à $6 \mathrm{~L} / \mathrm{min}$. Les intervalles d'incertitude ont été calculés selon la méthode du développement limité en série de Taylor de la dérivée totale (DOEBELIN, 1983). La fraction exportée du métolachlore est inférieure à celle de l'atrazine : ceci est dû au fait que, bien que les masses exportées soient voisines pour les deux herbicides, la masse de métolachlore appliquée a été deux fois supérieure à celle d'atrazine. Le Champ 1 présente des masses exportées très faibles. Les autres champs peuvent être comparés entre eux selon leur type de sol ou leurs pratiques culturales.

Tableau 5 Évaluation des masses totales de composés herbicides exportées par l'eau de drainage lors des deux événements pluviaux ${ }^{\mathrm{a}}$.

Table 5 Evaluation of the total masses of herbicides losses from drainage for the two rainfall events ${ }^{\mathrm{a}}$.

\begin{tabular}{|c|c|c|c|c|c|}
\hline \multirow{2}{*}{\multicolumn{2}{|c|}{$\begin{array}{l}\text { Exportation } \\
\text { des composés herbicides } \\
\text { (deux pluies) }\end{array}$}} & \multicolumn{4}{|c|}{ Champ agricole } \\
\hline & & $t$ & 3 & 5 & 6 \\
\hline \multicolumn{2}{|c|}{$\begin{array}{c}\text { Hauteur totale } \\
\text { d'eau drainée (mm) }\end{array}$} & 0,10 & $5-14$ & $2-5$ & $2-4$ \\
\hline \multirow{3}{*}{$\begin{array}{l}\text { Masse totale } \\
\text { exportée } \\
(\mathrm{mg} / \mathrm{ha})^{\mathrm{b}}\end{array}$} & Métolachlore & $0,1-0,5$ & $44-177$ & $12-51$ & $260-764$ \\
\hline & Atrazine & $0,3-0,4$ & $29-122$ & $14-61$ & $238-803$ \\
\hline & DEA & $0,3-0,5$ & 3-20 & $6-29$ & $57-226$ \\
\hline \multirow{2}{*}{$\begin{array}{c}\text { Fraction } \\
\text { exportée } \\
(\%)^{c}\end{array}$} & Métolachlore & $0,000-0,000$ & $0,002-0,007$ & $0,001-0,004$ & $0,013-0,039$ \\
\hline & Atrazine & $0,000-0,000$ & $0,002-0,010$ & $0,002-0,010$ & $0,025-0,083$ \\
\hline
\end{tabular}

a Excluant les Champs 2 et 4 dont les drains étaient submergés.

bébit du drain $x$ concentration. Les intervalles d'incertitude proviennent de l'erreur liée aux mesures de la concentration et du volume d'eau de drainage.

c Masse exportée/masse appliquée (en \%).

Labour avec application d'herbicides en surface totale. Les Champs 3 et 6 montrent que les masses exportées sont largement plus élevées pour le Champ 6. Ceci a été obtenu malgré un délai plus long entre l'application et les événements, ainsi qu'un contenu en sable pour le Champ 6 beaucoup moins élevé que celui du Champ 3 (tabl. 3). L'importante hauteur d'eau précipitée lors du second événement au Champ 6 (tabl. 3) serait en partie responsable des masses exportées plus élevées.

Application d'herbicides en surface totale et en bandes (sur labour). Les masses exportées pour le Champ 5 (bandes) sont très nettement inférieures à celles obtenues pour le Champ 3 (surface totale) : cette diminution est de 3,5 fois pour le métolachlore et de 2 fois pour l'atrazine. Sous des conditions pédo-climatiques semblables (tabl. 3), les masses exportées seraient donc nettement inférieures pour une application en bandes. La plus faible exportation pour le Champ 5 pourrait aussi être en partie attribuable au plus long délai entre l'application et les événements, comparativement au Champ 3 (tabl. 3). Par ailleurs, les Champs 4 
(bandes) et 3 (surface totale) sont adjacents et leurs conditions pédo-climatiques sont semblables (tabl. 3). Bien que les masses exportées n'aient pu être estimées pour le Champ 4, on retrouve des concentrations en herbicides environ 1,5 fois plus élevées avec l'application en surface totale, comparativement à celle en bandes. Enfin, il est pertinent de comparer l'exportation pour les Champs 5 (bandes) et 6 (surface totale). Les masses exportées pour le Champ 5 sont très nettement inférieures à celles obtenues pour le Champ 6 : cette diminution est de 1520 fois pour le métolachlore et de 13-17 fois pour l'atrazine. Dans les conditions rencontrées, les masses exportées sont très nettement inférieures suite à l'application en bandes. La faible exportation observée pour le Champ 5 pourrait être aussi en partie attribuable à un délai un peu plus long entre l'application et les événements, comparativement au Champ 6 (tabl. 3). Également, l'exportation plus élevée pour le Champ 6 pourrait avoir été causée en partie par l'importante hauteur d'eau précipitée lors du second événement (tabl. 3).

\subsubsection{Signification agro-environnementale}

Les pertes d'herbicides durant la saison de croissance sont minimes en cas de faible pluviométrie et d'assèchement des drains. Pour un sol argileux présentant un faible drainage et des événements pluviaux survenant un mois après l'application, les concentrations et les masses en herbicides sont très faibles. Pour des sols dont la texture varie de loam argileux à loam, la réponse des drains à une précipitation est relativement rapide (environ 6 à 12 heures) et les concentrations en herbicides peuvent atteindre 40-60 $\mu \mathrm{g} / \mathrm{L}$. Les concentrations ou les masses de métolachlore sont légèrement supérieures ou voisines à celles d'atrazine, ceci malgré une quantité appliquée deux fois supérieure à celle de l'atrazine. Ceci pourrait être attribué à : 1) une dégradation plus rapide du métolachlore, et 2) à une adsorption au sol du métolachlore $\left(K_{\mathrm{OC}}=200 \mathrm{~mL} / \mathrm{g}\right)$ plus importante que celle de l'atrazine $\left(K_{o c}=100 \mathrm{~mL} / \mathrm{g}\right)$. Notons que le destin des herbicides pourrait également être influencé par la composition des horizons inférieurs du profil de sol, composition qui peut différer de celle retrouvée en surface. Dans la majorité des cas, le RDA dans l'eau de drainage est supérieur à celui observé pour l'eau de ruissellement, ce qui illustre la dégradation de l'atrazine dans la zone non saturée. Pour des conditions pédo-climatiques peu différentes, l'application d'herbicides en bandes conduit à des masses exportées inférieures à celles obtenues avec l'application en surface totale. Toutefois, le délai entre l'application et les événements pluviaux, ainsi que les hauteurs d'eau précipitée peuvent varier d'un champ à l'autre, ce qui rend difficile l'évaluation de l'impact du seul mode-d'application des herbicides sur l'exportation. Lors des deux premiers événements et lorsqu'un ruissellement est observé, la fraction d'herbicides exportée par drainage semble être bien inférieure à celle exportée par ruissellement.

\section{4- CONCLUSION}

L'étude a évalué, sur six champs agricoles et pour une saison de culture, l'exportation d'herbicides vers les eaux de surface par ruissellement et par drainage, ceci pour des conditions pédo-climatiques et agronomiques représentati- 
ves de la culture intensive du maïs-grain au Québec. Seulement deux champs ont présenté un ruissellement quittant le champ, avec des concentrations atteignant $1200 \mu \mathrm{g} / \mathrm{L}$ et $2400 \mu \mathrm{g} / \mathrm{L}$ respectivement lors des deux événements pluviaux d'importance ayant suivi l'application des herbicides. Lors du premier événement, les concentrations mesurées dans le cours d'eau récepteur à proximité d'un champ dépassaient les critères canadiens de qualité pour la vie aquatique. Pour les conditions rencontrées, la charge exportée en herbicides serait inférieure dans le cas du semis direct, comparativement au labour. Pour l'eau de drainage, l'exportation est notamment fonction de la texture du sol, des hauteurs d'eau de précipitation (et drainée) et du délai entre l'application et les événements pluviaux. Pour quatre champs, les concentrations sont généralement inférieures à 1-2 $\mu \mathrm{g} / \mathrm{L}$. Deux champs ont montré des concentrations atteignant $40-$ $60 \mu \mathrm{g} / \mathrm{L}$. La charge exportée serait inférieure dans le cas de l'application d'herbicides en bandes, comparativement à l'application en surface totale.

Lors des deux événements pluviaux, le volume d'eau de ruissellement a été limité pour ces champs. Cependant, la fraction d'herbicides exportée par ruissellement (telle qu'estimée à partir de coefficients de ruissellement probables) serait bien supérieure à celle quantifiée pour le drainage. Ainsi, et en complémentarité avec le travail de conservation du sol et l'application des herbicides en bandes, des mesures préventives telles que l'établissement de dispositifs enherbés sur les rives des cours d'eau (bandes riveraines) seraient susceptibles de diminuer l'importance de l'exportation d'herbicides par ruissellement. Des études de suivi, menés comme dans la présente étude sur plusieurs champs agricoles mais durant d'autres saisons de croissance, permettraient d'estimer la variabilité interannuelle des charges exportées en herbicides. De telles études contribueraient à confirmer l'importance relative des pertes d'herbicides par ruissellement et par drainage.

\section{REMERCIEMENTS}

Les auteurs remercient MM Florian Bernard et Marc Trudelle (F. BERNARD Inc.) pour l'identification des sites d'étude et pour la supervision des activités agricoles, de même que les agriculteurs participants. Ce projet a bénéficié du support financier du programme FRDTE du ministère de l'Environnement et de la Faune du Québec, ainsi que du Conseil de recherches en sciences naturelles et en génie du Canada.

\section{RÉFÉRENCES BIBLIOGRAPHIQUES}

ADAMS, C.D., THURMAN, E.M. (1991). Formation and transport of deethylatrazine in the soil and vadose zone. J. Environ. Qual., $20: 540-547$.
AYOTTE, P., LARUE, M. (1990). Micropolluants organiques, Campagnes d'échantillonnage printemps/été 1987 et hiver 1988. Ministère de l'Environnement du 
Québec, Direction des écosystèmes urbains, $178 \mathrm{p}$.

BERNARD, C., LAVERDIĖRE, M.R., WICHEREK, S. (1996). Agricultural and environmental efficiency of conservation practices. Proc. 2nd Int. Cong. Europ. Soc. for Soil Conservation, September 1-7, Freising, Germany, p. 73.

BERRYMAN, D., GIROUX, I. (1994). La contamination des cours d'eau par les pesticides dans les régions de culture intensive du maïs au Québec. Campagnes d'échantillonnage de 1992 et 1993. Ministère de l'Environnement et de la Faune du Québec, Direction des écosystèmes aquatiques, Envirodoq EN940594, rapport $n^{\circ}$ PES-4, $134 \mathrm{p}$.

CANN, D.B., LAJOIE, P., STOBBE, P.C. (1947). Soil survey of Shefford, Brome and Missisquoi counties in the Province of Quebec. Experimental Farm Service. Dominion Department of Agriculture. Quebec Department of Agriculture and MacDonald College of McGill University. $84 \mathrm{p}$.

CEPPAC Comité d'experts sur la prospection pédologique d'Agriculture Canada (1987). Le système canadien de classification des sols. Direction générale de la recherche. Agriculture Canada. Publication 1646 , $170 \mathrm{p}$.

CHESTERS, G., SIMSIMAN, G.V., LEVY, J., ALHAJJAR, B.J., FATHULLA, R.N., HARKIN, J.M. (1989). Environmental fate of alachlor and metolachlor. Rev. Environ. Contam. Toxicol., $110: 1.74$.

CPVQ Conseil des productions végétales du Québec. Méthodes d'analyse des sols, des fumiers et des tissus végétaux (1988). Publication AGDEX 533.

DOEBELIN, E.O. (1983). Measurement systems. Applications and design. McGrawHill Book Co., Third edition, $876 \mathrm{p}$.

EADIE, A.G., SWANTON, C.J., SHAW, J.E. (1992). Banded herbicide applications and cultivation in a modified nc-till corn (Zea mays L.) system. Weed Technol., 6 : 535-542.

FAWCETT, R.S., CHRISTENSEN, B.R., TIERNEY, D.P. (1994). The impact of conservation tillage on pesticide runoff into surface water: A review and analysis. $J$. Soil Water Conserv., $49: 126-135$.
FLURY, M. (1996). Experimental evidence of transport of pesticides through field soils a review. J. Environ. Qual., 25 : 25-45.

FORREST, S., CAUX, P.Y. (1990). Pesticides in tributaries of the St-Laurence River 1987-1988-Program Report. Centre Saint-Laurent, Environment Canada, $139 \mathrm{p}$.

GAYNOR, J.D., TAN, C.S., DRURY, C.F., VAN WESENBEECK, I.J., WELACKY, T.W. (1995a). Atrazine in surface and subsurface runoff as affected by cultural practices. Water Qual. Res. J. Canada, 30 (3) : 513-531.

GAYNOR, J.D., MacTAVISH, D.C., FINDLAY, W.I. (1995b). Atrazine and metolachlor loss in surface and subsurface runoff from three tillage treatments in corn. J. Environ. Qual., 24 : 246-256.

GAYNOR, J.D., MacTAVISH, D.C., FINDLAY, W.I. (1992). Surface and subsurface transport of atrazine and alachlor from a Brookston clay loam under continuous corn production. Arch. Environ. Contam. Toxicol., $23: 240-245$.

KAY, R.L., BAKER, J.L. (1989). Management with ridge tillage to reduce chemical losses. Trans. ASAE, paper $n^{\circ}$ 89-2157.

LAFRANCE, P., BANTON, O. (1996). Evaluation in situ de l'impact de pratiques culturales sur la persistance et l'exportation d'herbicides. Actes du Seminaire national Hydrosystèmes - Groupe Français des Pesticides : "Processus de transfert des produits phytosanitaires et modélisation dans les bassins versants ". 22-23 mai 1996, Nancy, France, CEMAGREF Éditions, Antony, France, pp. 227-235.

LAFRANCE, P., BANTON, O., F. BERNARD Inc. (1996). Évaluation environnementale des pratiques culturales sur maïs pour la réduction des pertes d'herbicides. INRSEau, rapport de recherche $n^{\circ} \mathrm{R}-390$ pour la Direction de l'information environnementale et de la recherche, ministère de l'Environnement et de la Faune du Québec, $919 \mathrm{p}$.

LAMONTAGNE, L., NOLIN, M.C. (1990). Étude pédologique du comté de Verchères (Québec). Équipe pédologique du Québec. Centre de recherches sur les terres, Agriculture Canada, Sainte-Foy, Québec. Contribution no 87-92-1990. 287 p. 
LEBLANC, M.L., ClOUTIER, D.C., LEROUX, G.D. (1995). Réduction de l'utilisation des herbicides dans le maïs-grain par une application d'herbicides en bandes combinée à des sarclages mécaniques. Weed Res., $35: 511-522$.

LEONARD, R.A. (1990). Movement of pesticides into surface waters. Dans : Pesticides in the soil environment: Processes, impacts, and modeling, H.H. Cheng (ed.), Soil Science Society of America, Madison, WI, USA, pp. 303-350.

PESANT, A.R., DIONNE, J.L., GENEST, J. (1987). Soil and nutrient losses in surface runoff from conventional and no-till corn systems. Can. J. Soil Sci., 67 : 835-843.

TOPP, E., SMITH, W. (1992). Sorption of the herbicides atrazine and metolachlor to selected plastics and silicone rubber. $J$. Environ. Qual., 21 : 316-317.

THURMAN, E.M., GOOLSBY, D.A., MEYER, M.T., MILLS, M.S., POMES, M.L., KOLPIN, D.W. (1992). A reconnaissance study of herbicides and their metabolites in surface water of the Midwestern United States using immunoassay and gas chromatography/mass spectrometry. Environ, Sci. Technol., 26 : 2440-2447. 